\title{
Higgs boson searches in CP-conserving and CP-violating MSSM scenarios with the DELPHI detector
}

\section{The DELPHI Collaboration}

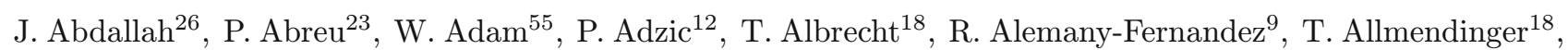
P.P. Allport ${ }^{24}$, U. Amaldi ${ }^{30}$, N. Amapane ${ }^{48}$, S. Amato ${ }^{52}$, E. Anashkin ${ }^{37}$, A. Andreazza ${ }^{29}$, S. Andringa ${ }^{23}$, N. Anjos ${ }^{23}$, P. Antilogus ${ }^{26}$, W-D. Apel ${ }^{18}$, Y. Arnoud ${ }^{15}$, S. Ask ${ }^{27}$, B. Asman 4 , J.E. Augustin ${ }^{26}$, A. Augustinus ${ }^{9}$, P. Baillon ${ }^{9}$, A. Ballestrero ${ }^{49}$, P. Bambade ${ }^{21}$, R. Barbier ${ }^{28}$, D. Bardin ${ }^{17}$, G.J. Barker ${ }^{57}$, A. Baroncelli ${ }^{40}$, M. Battaglia ${ }^{9}$, M. Baubillier ${ }^{26}$, K-H. Becks ${ }^{58}$, M. Begalli ${ }^{7}$, A. Behrmann ${ }^{58}$, E. Ben-Haim ${ }^{21}$, N. Benekos ${ }^{33}$, A. Benvenuti ${ }^{5}$, C. Berat ${ }^{15}$, M. Berggren ${ }^{26}$, L. Berntzon ${ }^{47}$, D. Bertrand ${ }^{2}$, M. Besancon ${ }^{41}$, N. Besson ${ }^{41}$, D. Bloch $^{10}$, M. Blom $^{32}$, M. Bluj ${ }^{56}$, M. Bonesini ${ }^{30}$, M. Boonekamp ${ }^{41}$, P.S.L. Booth ${ }^{24} \dagger$, G. Borisov ${ }^{22}$, O. Botner ${ }^{53}$, B. Bouquet $^{21}$, T.J.V. Bowcock ${ }^{24}$, I. Boyko ${ }^{17}$, M. Bracko ${ }^{44}$, R. Brenner ${ }^{53}$, E. Brodet ${ }^{36}$, P. Bruckman ${ }^{19}$, J.M. Brunet ${ }^{8}$, B. Buschbeck ${ }^{55}$, P. Buschmann ${ }^{58}$, M. Calvi ${ }^{30}$, T. Camporesi ${ }^{9}$, V. Canale ${ }^{39}$, F. Carena ${ }^{9}$, N. Castro ${ }^{23}$, F. Cavallo ${ }^{5}$, M. Chapkin ${ }^{43}$, Ph. Charpentier ${ }^{9}$, P. Checchia ${ }^{37}$, R. Chierici ${ }^{9}$, P. Chliapnikov ${ }^{43}$, J. Chudoba ${ }^{9}$, S.U. Chung ${ }^{9}$, K. Cieslik ${ }^{19}$, P. Collins ${ }^{9}$, R. Contri ${ }^{14}$, G. Cosme ${ }^{21}$, F. Cossutti ${ }^{50}$, M.J. Costa ${ }^{54}$, D. Crennell ${ }^{38}$, J. Cuevas ${ }^{35}$, J. D'Hondt ${ }^{2}$, J. Dalmau ${ }^{47}$, T. da Silva ${ }^{52}$, W. Da Silva ${ }^{26}$, G. Della Ricca ${ }^{50}$, A. De Angelis ${ }^{51}$, W. De Boer ${ }^{18}$, C. De Clercq ${ }^{2}$, B. De Lotto ${ }^{51}$, N. De Maria ${ }^{48}$, A. De Min ${ }^{37}$, L. de Paula ${ }^{52}$, L. Di Ciaccio ${ }^{39}$, A. Di Simone ${ }^{40}$, K. Doroba ${ }^{56}$, J. Drees ${ }^{58,9}$, G. Eigen ${ }^{4}$, T. Ekelof ${ }^{53}$, M. Ellert ${ }^{53}$, M. Elsing ${ }^{9}$, M.C. Espirito Santo ${ }^{23}$, G. Fanourakis ${ }^{12}$, D. Fassouliotis ${ }^{12,3}$, M. Feindt ${ }^{18}$, J. Fernandez ${ }^{42}$, A. Ferrer ${ }^{54}$, F. Ferro ${ }^{14}$, U. Flagmeyer ${ }^{58}$, H. Foeth ${ }^{9}$, E. Fokitis ${ }^{33}$, F. Fulda-Quenzer ${ }^{21}$, J. Fuster ${ }^{54}$, M. Gandelman ${ }^{52}$, C. Garcia ${ }^{54}$, Ph. Gavillet ${ }^{9}$, E. Gazis ${ }^{33}$, R. Gokieli ${ }^{9,56}$, B. Golob ${ }^{44} 46$, G. Gomez-Ceballos ${ }^{42}$, P. Goncalves ${ }^{23}$, E. Graziani ${ }^{40}$, G. Grosdidier ${ }^{21}$, K. Grzelak ${ }^{56}$, J. Guy ${ }^{38}$, C. Haag $^{18}$, A. Hallgren ${ }^{53}$, K. Hamacher ${ }^{58}$, K. Hamilton ${ }^{36}$, S. Haug ${ }^{34}$, F. Hauler ${ }^{18}$, V. Hedberg ${ }^{27}$, M. Hennecke ${ }^{18}$, H. Herr ${ }^{9, \dagger}$, J. Hoffman ${ }^{56}$, S-O. Holmgren ${ }^{47}$, P.J. Holt ${ }^{9}$, M.A. Houlden ${ }^{24}$, J.N. Jackson ${ }^{24}$, G. Jarlskog ${ }^{27}$, P. Jarry ${ }^{41}$, D. Jeans ${ }^{36}$, E.K. Johansson ${ }^{47}$, P.D. Johansson ${ }^{47}$, P. Jonsson ${ }^{28}$, C. Joram ${ }^{9}$, L. Jungermann ${ }^{18}$, F. Kapusta ${ }^{26}$, S. Katsanevas ${ }^{28}$, E. Katsoufis ${ }^{33}$, G. Kernel ${ }^{44}$, B.P. Kersevan ${ }^{44}{ }^{46}$, U. Kerzel ${ }^{18}$, B.T. King ${ }^{24}$, N.J. Kjaer ${ }^{9}$, P. Kluit ${ }^{32}$, P. Kokkinias ${ }^{12}$, C. Kourkoumelis ${ }^{3}$, O. Kouznetsov ${ }^{17}$, Z. Krumstein ${ }^{17}$, M. Kucharczyk ${ }^{19}$, J. Lamsa ${ }^{1}$, G. Leder ${ }^{55}$, F. Ledroit ${ }^{15}$, L. Leinonen ${ }^{47}$, R. Leitner ${ }^{31}$, J. Lemonne ${ }^{2}$, V. Lepeltier ${ }^{21}$, T. Lesiak ${ }^{19}$, W. Liebig ${ }^{58}$, D. Liko ${ }^{55}$, A. Lipniacka ${ }^{47}$, J.H. Lopes ${ }^{52}$, J.M. Lopez ${ }^{35}$, D. Loukas ${ }^{12}$, P. Lutz ${ }^{41}$, L. Lyons ${ }^{36}$, J. MacNaughton ${ }^{55}$, A. Malek ${ }^{58}$, S. Maltezos ${ }^{33}$, F. Mandl ${ }^{55}$, J. Marco ${ }^{42}$, R. Marco ${ }^{42}$, B. Marechal ${ }^{52}$, M. Margoni ${ }^{37}$, J-C. Marin ${ }^{9}$, C. Mariotti ${ }^{9}$, A. Markou ${ }^{12}$, C. Martinez-Rivero ${ }^{42}$, J. Masik ${ }^{13}$, N. Mastroyiannopoulos ${ }^{12}$, F. Matorras ${ }^{42}$, C. Matteuzzi ${ }^{30}$, F. Mazzucato ${ }^{37}$, M. Mazzucato ${ }^{37}$, R. McNulty ${ }^{24}$, C. Meroni ${ }^{29}$, E. Migliore ${ }^{48}$, W. Mitaroff ${ }^{55}$, U. Mjoernmark ${ }^{27}$, T. Moa ${ }^{47}$, M. Moch ${ }^{18}$, K. Moenig ${ }^{9,11}$, R. Monge ${ }^{14}$, J. Montenegro ${ }^{32}$, D. Moraes ${ }^{52}$, S. Moreno ${ }^{23}$, P. Morettini ${ }^{14}$, U. Mueller ${ }^{58}$, K. Muenich ${ }^{58}$, M. Mulders ${ }^{32}$, L. Mundim ${ }^{7}$, W. Murray ${ }^{38}$, B. Muryn ${ }^{20}$, G. Myatt ${ }^{36}$, T. Myklebust ${ }^{34}$, M. Nassiakou ${ }^{12}$, F. Navarria ${ }^{5}$, K. Nawrocki ${ }^{56}$, R. Nicolaidou ${ }^{41}$, M. Nikolenko ${ }^{17,10}$, A. Oblakowska-Mucha ${ }^{20}$, V. Obraztsov ${ }^{43}$, A. Olshevski ${ }^{17}$, A. Onofre ${ }^{23}$, R. Orava ${ }^{16}$, K. Osterberg ${ }^{16}$, A. Ouraou ${ }^{41}$, A. Oyanguren ${ }^{54}$, M. Paganoni ${ }^{30}$, S. Paiano ${ }^{5}$, J.P. Palacios ${ }^{24}$, H. Palka ${ }^{19}$, Th.D. Papadopoulou ${ }^{33}$, L. Pape ${ }^{9}$, C. Parkes ${ }^{25}$, F. Parodi ${ }^{14}$, U. Parzefall ${ }^{9}$, A. Passeri ${ }^{40}$, O. Passon ${ }^{58}$, L. Peralta ${ }^{23}$, V. Perepelitsa ${ }^{54}$, A. Perrotta ${ }^{5}$, A. Petrolini ${ }^{14}$, J. Piedra ${ }^{42}$, L. Pieri ${ }^{40}$, F. Pierre ${ }^{41}$, M. Pimenta ${ }^{23}$, E. Piotto ${ }^{9}$, T. Podobnik ${ }^{44}$, , V. Poireau $^{9}$, M.E. Pol ${ }^{6}$, G. Polok ${ }^{19}$, V. Pozdniakov ${ }^{17}$, N. Pukhaeva ${ }^{17}$, A. Pullia ${ }^{30}$, J. Rames ${ }^{13}$, A. Read ${ }^{34}$, P. Rebecchi ${ }^{9}$, J. Rehn ${ }^{18}$, D. Reid $^{32}$, R. Reinhardt ${ }^{58}$, P. Renton ${ }^{36}$, F. Richard ${ }^{21}$, J. Ridky ${ }^{13}$, M. Rivero ${ }^{42}$, D. Rodriguez ${ }^{42}$, A. Romero ${ }^{48}$, P. Ronchese $^{37}$, P. Roudeau ${ }^{21}$, T. Rovelli ${ }^{5}$, V. Ruhlmann-Kleider ${ }^{41}$, D. Ryabtchikov ${ }^{43}$, A. Sadovsky ${ }^{17}$, L. Salmi ${ }^{16}$, J. Salt ${ }^{54}$, C. Sander ${ }^{18}$, A. Savoy-Navarro ${ }^{26}$, U. Schwickerath ${ }^{9}$, R. Sekulin ${ }^{38}$, M. Siebel ${ }^{58}$, A. Sisakian ${ }^{17}$, G. Smadja ${ }^{28}$, O. Smirnova ${ }^{27}$, A. Sokolov ${ }^{43}$, A. Sopczak ${ }^{22}$, R. Sosnowski ${ }^{56}$, T. Spassov ${ }^{9}$, M. Stanitzki ${ }^{18}$, A. Stocchi ${ }^{21}$, J. Strauss ${ }^{55}$, B. Stugu ${ }^{4}$, M. Szczekowski ${ }^{56}$, M. Szeptycka ${ }^{56}$, T. Szumlak ${ }^{20}$, T. Tabarelli ${ }^{30}$, A.C. Taffard ${ }^{24}$, F. Tegenfeldt ${ }^{53}$, J. Timmermans ${ }^{32, \text { a }}$, L. Tkatchev ${ }^{17}$, M. Tobin ${ }^{24}$, S. Todorovova ${ }^{13}$, B. Tome ${ }^{23}$, A. Tonazzo ${ }^{30}$, P. Tortosa ${ }^{54}$, P. Travnicek ${ }^{13}$, D. Treille ${ }^{9}$, G. Tristram ${ }^{8}$, M. Trochimczuk ${ }^{56}$, C. Troncon ${ }^{29}$, M-L. Turluer ${ }^{41}$, I.A. Tyapkin ${ }^{17}$, P. Tyapkin ${ }^{17}$, S. Tzamarias ${ }^{12}$, V. Uvarov ${ }^{43}$, G. Valenti ${ }^{5}$, P. Van Dam ${ }^{32}$, J. Van Eldik ${ }^{9}$, N. van Remortel ${ }^{16}$, I. Van Vulpen ${ }^{9}$, G. Vegni ${ }^{29}$, F. Veloso ${ }^{23}$, W. Venus ${ }^{38}$, P. Verdier $^{28}$, V. Verzi ${ }^{39}$, D. Vilanova ${ }^{41}$, L. Vitale $^{50}$, V. Vrba $^{13}$, H. Wahlen ${ }^{58}$, A.J. Washbrook ${ }^{24}$, C. Weiser ${ }^{18}$, D. Wicke ${ }^{9}$, J. Wickens ${ }^{2}$, G. Wilkinson ${ }^{36}$, M. Winter ${ }^{10}$, M. Witek ${ }^{19}$, O. Yushchenko ${ }^{43}$, A. Zalewska ${ }^{19}$, P. Zalewski ${ }^{56}$, D. Zavrtanik ${ }^{45}$, V. Zhuravlov ${ }^{17}$, N.I. Zimin ${ }^{17}$, A. Zintchenko ${ }^{17}$, M. Zupan ${ }^{12}$ 
1 Department of Physics and Astronomy, Iowa State University, Ames IA 50011-3160, USA

2 IIHE, ULB-VUB, Pleinlaan 2, 1050 Brussels, Belgium

3 Physics Laboratory, University of Athens, Solonos Str. 104, 10680 Athens, Greece

4 Department of Physics, University of Bergen, Allégaten 55, 5007 Bergen, Norway

5 Dipartimento di Fisica, Università di Bologna and INFN, Via Irnerio 46, 40126 Bologna, Italy

${ }^{6}$ Centro Brasileiro de Pesquisas Físicas, rua Xavier Sigaud 150, 22290 Rio de Janeiro, Brazil

7 Inst. de Física, Univ. Estadual do Rio de Janeiro, rua São Francisco Xavier 524, Rio de Janeiro, Brazil

8 Collège de France, Lab. de Physique Corpusculaire, IN2P3-CNRS, 75231 Paris Cedex 05, France

${ }^{9}$ CERN, 1211 Geneva 23, Switzerland

10 Institut de Recherches Subatomiques, IN2P3-CNRS/ULP-BP20, 67037 Strasbourg Cedex, France

11 Now at DESY-Zeuthen, Platanenallee 6, 15735 Zeuthen, Germany

12 Institute of Nuclear Physics, N.C.S.R. Demokritos, P.O. Box 60228, 15310 Athens, Greece

13 FZU, Inst. of Phys. of the C.A.S. High Energy Physics Division, Na Slovance 2, 180 40, Praha 8, Czech Republic

14 Dipartimento di Fisica, Università di Genova and INFN, Via Dodecaneso 33, 16146 Genova, Italy

15 Institut des Sciences Nucléaires, IN2P3-CNRS, Université de Grenoble 1, 38026 Grenoble Cedex, France

16 Helsinki Institute of Physics and Department of Physical Sciences, P.O. Box 64, 00014 University of Helsinki, Finland

17 Joint Institute for Nuclear Research, Dubna, Head Post Office, P.O. Box 79, 101000 Moscow, Russian Federation

18 Institut für Experimentelle Kernphysik, Universität Karlsruhe, Postfach 6980, 76128 Karlsruhe, Germany

19 Institute of Nuclear Physics PAN,Ul. Radzikowskiego 152, 31142 Krakow, Poland

${ }^{20}$ Faculty of Physics and Nuclear Techniques, University of Mining and Metallurgy, 30055 Krakow, Poland

${ }^{21}$ Université de Paris-Sud, Lab. de l'Accélérateur Linéaire, IN2P3-CNRS, Bât. 200, 91405 Orsay Cedex, France

22 School of Physics and Chemistry, University of Lancaster, Lancaster LA1 4YB, UK

${ }^{23}$ LIP, IST, FCUL - Av. Elias Garcia, 14-1 ${ }^{\circ}$, 1000 Lisboa Codex, Portugal

24 Department of Physics, University of Liverpool, P.O. Box 147, Liverpool L69 3BX, UK

25 Dept. of Physics and Astronomy, Kelvin Building, University of Glasgow, Glasgow G12 8QQ, UK

${ }^{26}$ LPNHE, IN2P3-CNRS, Univ. Paris VI et VII, Tour 33 (RdC), 4 place Jussieu, 75252 Paris Cedex 05, France

27 Department of Physics, University of Lund, Sölvegatan 14, 22363 Lund, Sweden

28 Université Claude Bernard de Lyon, IPNL, IN2P3-CNRS, 69622 Villeurbanne Cedex, France

29 Dipartimento di Fisica, Università di Milano and INFN-MILANO, Via Celoria 16, 20133 Milan, Italy

30 Dipartimento di Fisica, Univ. di Milano-Bicocca and INFN-MILANO, Piazza della Scienza 3, 20126 Milan, Italy

31 IPNP of MFF, Charles Univ., Areal MFF, V Holesovickach 2, 18000, Praha 8, Czech Republic

32 NIKHEF, Postbus 41882, 1009 DB Amsterdam, The Netherlands

33 National Technical University, Physics Department, Zografou Campus, 15773 Athens, Greece

34 Physics Department, University of Oslo, Blindern, 0316 Oslo, Norway

35 Dpto. Fisica, Univ. Oviedo, Avda. Calvo Sotelo s/n, 33007 Oviedo, Spain

36 Department of Physics, University of Oxford, Keble Road, Oxford OX1 3RH, UK

37 Dipartimento di Fisica, Università di Padova and INFN, Via Marzolo 8, 35131 Padua, Italy

38 Rutherford Appleton Laboratory, Chilton, Didcot OX11 OQX, UK

39 Dipartimento di Fisica, Università di Roma II and INFN, Tor Vergata, 00173 Rome, Italy

40 Dipartimento di Fisica, Università di Roma III and INFN, Via della Vasca Navale 84, 00146 Rome, Italy

41 DAPNIA/Service de Physique des Particules, CEA-Saclay, 91191 Gif-sur-Yvette Cedex, France

42 Instituto de Fisica de Cantabria (CSIC-UC), Avda. los Castros s/n, 39006 Santander, Spain

43 Inst. for High Energy Physics, Serpukov P.O. Box 35, Protvino, (Moscow Region), Russian Federation

44 J. Stefan Institute, Jamova 39, 1000 Ljubljana, Slovenia

45 Laboratory for Astroparticle Physics, University of Nova Gorica, Kostanjeviska 16a, 5000 Nova Gorica, Slovenia

46 Department of Physics, University of Ljubljana, 1000 Ljubljana, Slovenia

47 Fysikum, Stockholm University, Box 6730, 11385 Stockholm, Sweden

48 Dipartimento di Fisica Sperimentale, Università di Torino and INFN, Via P. Giuria 1, 10125 Turin, Italy

49 INFN, Sezione di Torino and Dipartimento di Fisica Teorica, Università di Torino, Via Giuria 1, 10125 Turin, Italy

50 Dipartimento di Fisica, Università di Trieste and INFN, Via A. Valerio 2, 34127 Trieste, Italy

51 Istituto di Fisica, Università di Udine and INFN, 33100 Udine, Italy

52 Univ. Federal do Rio de Janeiro, C.P. 68528 Cidade Univ., Ilha do Fundão 21945-970 Rio de Janeiro, Brazil

53 Department of Radiation Sciences, University of Uppsala, P.O. Box 535, 75121 Uppsala, Sweden

54 IFIC, Valencia-CSIC, and D.F.A.M.N., U. de Valencia, Avda. Dr. Moliner 50, 46100 Burjassot (Valencia), Spain

55 Institut für Hochenergiephysik, Österr. Akad. d. Wissensch., Nikolsdorfergasse 18, 1050 Vienna, Austria

56 Inst. Nuclear Studies and University of Warsaw, Ul. Hoza 69, 00681 Warsaw, Poland

57 Now at University of Warwick, Coventry CV4 7AL, UK

58 Fachbereich Physik, University of Wuppertal, Postfach 100 127, 42097 Wuppertal, Germany

$\dagger$ deceased 
Erratum to:

Eur. Phys. J. C 54, 1-35 (2008)

DOI 10.1140/epjc/s10052-007-0506-1
In the original version of this article unfortunatly the bottom-right plots in Figs. 6-8 and 10 were reproduced wrongly (on pages 14, 15, 16 and 18). The corrected figures are given below.
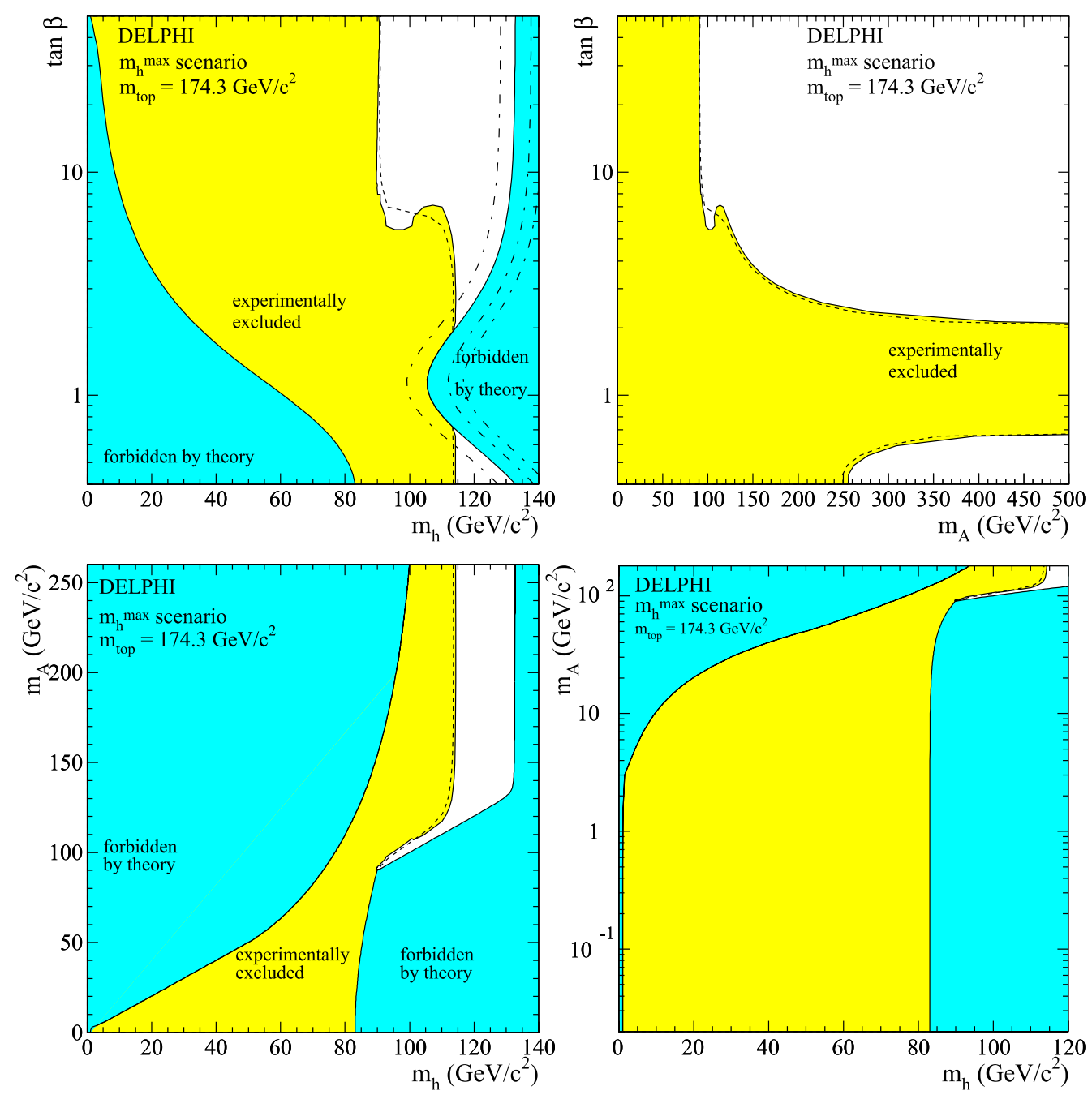

Fig. 6. MSSM $m_{h}^{\max }$ scenario for a top mass of $174.3 \mathrm{GeV} / c^{2}$ : regions excluded at $95 \%$ CL by combining the results of the Higgs boson searches in the whole DELPHI data sample (light-grey). The dashed curves show the median expected limits. The mediumgrey areas are the regions not allowed by theory. The dash-dotted lines in the top left-hand plot are the theoretical upper bounds for a top mass of $169.2,179.4$ and $183.0 \mathrm{GeV} / c^{2}$ (from left to right)

The online version of the original article can be found at http://dx.doi.org/10.1140/epjc/s10052-007-0506-1.

\footnotetext{
a e-mail: Jan.Timmermans@cern.ch
} 

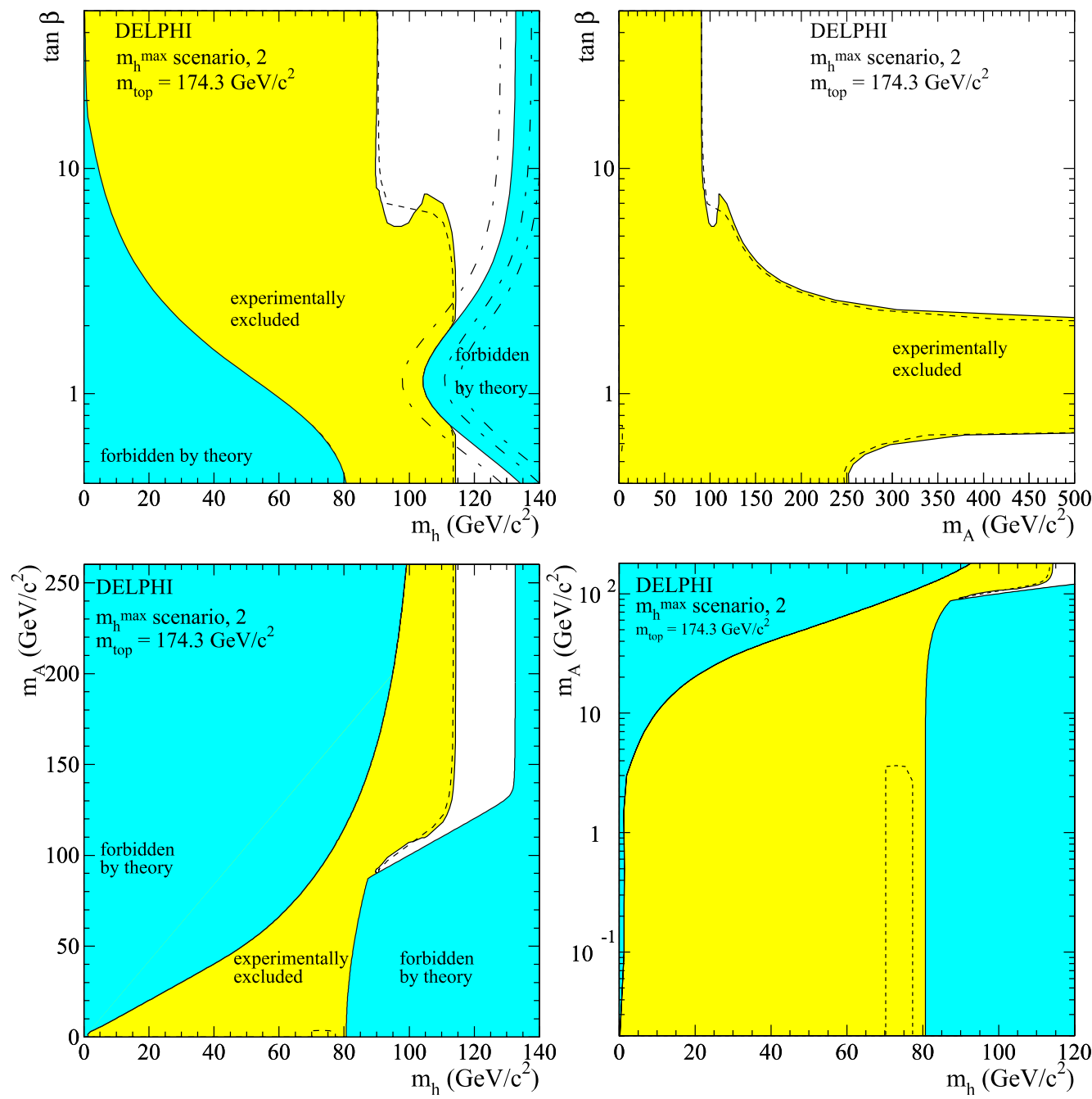

Fig. 7. MSSM $m_{h}^{\max }$ scenario with positive $\mu$ for a top mass of $174.3 \mathrm{GeV} / c^{2}$ : regions excluded at $95 \%$ CL by combining the results of the Higgs boson searches in the whole DELPHI data sample (light-grey). The dashed curves show the median expected limits. The medium-grey areas are the regions not allowed by theory. The dash-dotted lines in the top left-hand plot are the theoretical upper bounds for a top mass of $169.2,179.4$ and $183.0 \mathrm{GeV} / c^{2}$ (from left to right) 

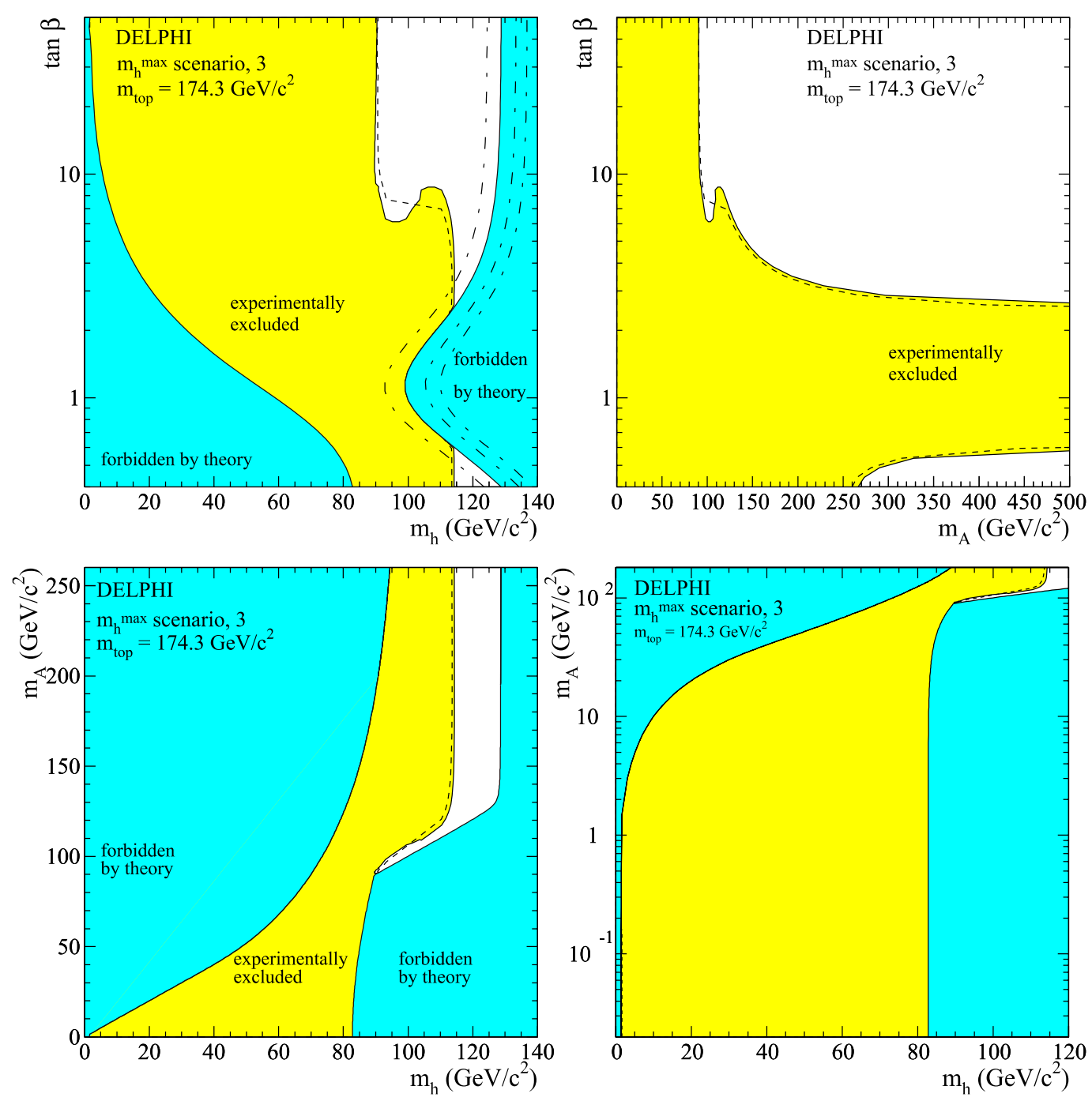

Fig. 8. MSSM $m_{h}^{\max }$ scenario with positive $\mu$ and negative $X_{t}$ for a top mass of $174.3 \mathrm{GeV} / c^{2}:$ regions excluded at $95 \%$ CL by combining the results of the Higgs boson searches in the whole DELPHI data sample (light-grey). The dashed curves show the median expected limits. The medium-grey areas are the regions not allowed by theory. The dash-dotted lines in the top left-hand plot are the theoretical upper bounds for a top mass of $169.2,179.4$ and $183.0 \mathrm{GeV} / c^{2}($ from left to right) 

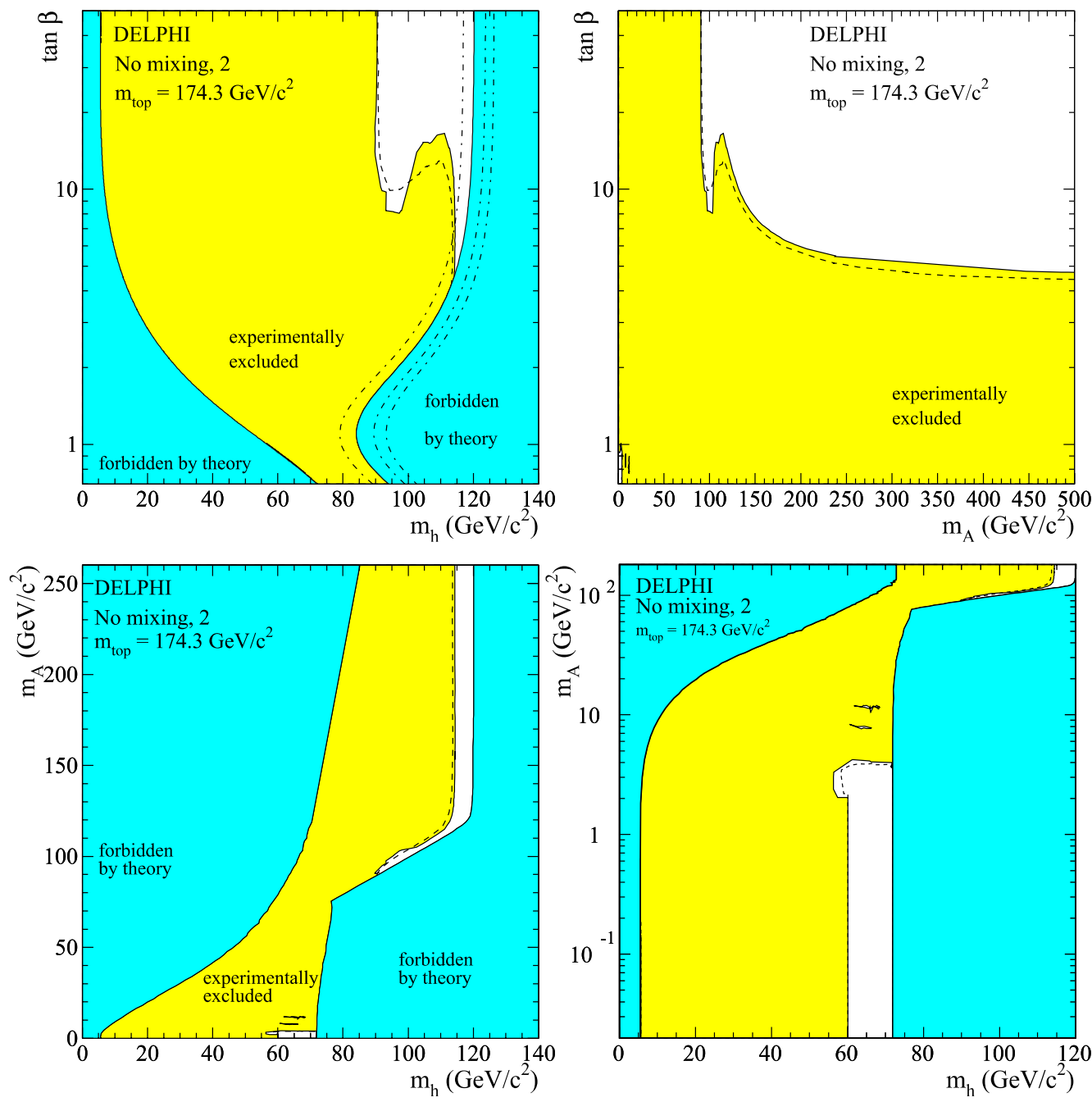

Fig. 10. MSSM no mixing scenario with positive $\mu$ and large $M_{\text {susy }}$ for a top mass of $174.3 \mathrm{GeV} / c^{2}$ : regions excluded at $95 \%$ CL by combining the results of the Higgs boson searches in the whole DELPHI data sample (light-grey). Among the four unexcluded regions at low $m_{A}$, the strip at low $m_{H}$ is fully excluded by the limit on the $Z$ partial width that would be due to new physics [40]. The dashed curves show the median expected limits. The medium-grey areas are the regions not allowed by theory. The dashdotted lines in the top left-hand plot are the theoretical upper bounds for a top mass of $169.2,179.4$ and $183.0 \mathrm{GeV} / c^{2}$ (from left to right) 